\title{
Efficiency and Duality in Nondifferentiable Multiobjective Programming Involving Directional Derivative
}

\author{
Izhar Ahmad \\ Department of Mathematics and Statistics, King Fahd University of Petroleum and Minerals, \\ Saudi Arabia, India \\ E-mail: izharmaths@hotmail.com; drizhar@kfupm.edu.sa \\ Received December 24, 2010; revised February 24, 2011; accepted February 26, 2011
}

\begin{abstract}
In this paper, we introduce a new class of generalized $d_{1}$-univexity in which each component of the objective and constraint functions is directionally differentiable in its own direction $\mathrm{d}_{\mathrm{i}}$ for a nondifferentiable multiobjective programming problem. Based upon these generalized functions, sufficient optimality conditions are established for a feasible point to be efficient and properly efficient under the generalised $\mathrm{d}_{\mathrm{I}}$-univexity requirements. Moreover, weak, strong and strict converse duality theorems are also derived for Mond-Weir type dual programs.
\end{abstract}

Keywords: Multiobjective Programming, Nondifferentiable Programming, Generalized $\mathrm{d}_{\mathrm{I}}$-Univexity, Sufficiency, Duality

\section{Introduction}

The field of multiobjective programming, also known as vector programming, has grown remarkably in different directions in the setting of optimality conditions and duality theory. It has been enriched by the applications of various types of generalizations of convexity theory, with and without differentiability assumptions, and in the framework of continuous time programming, fractional programming, inverse vector optimization, saddle point theory, symmetric duality and vector variational inequalities etc.

Hanson [1] introduced a class of functions by generalizing the difference vector $\boldsymbol{x}-\overline{\boldsymbol{x}}$ in the definition of a convex function to any vector function $\eta(\boldsymbol{x}, \overline{\boldsymbol{x}})$. These functions were named invex by Craven [2] and $\eta$-convex by Kaul and Kaur [3]. Hanson and Mond [4] defined two new classes of functions called Type I and Type II functions, which were further generalized to pseudo Type I and quasi Type I functions by Rueda and Hanson [5]. Zhao [6] established optimality conditions and duality in nonsmooth scalar programming problems assuming Clarke [7] generalized subgradients under Type I functions.

Kaul et al. [8] extended the concept of type I and its generalizations for a multiobjective programming problem. They investigated optimality conditions and derived Wolfe type and Mond-Weir type duality results. Suneja and Srivastava [9] introduced generalized d-type I functions in terms of directional derivative for a multiobjective programming problem and discussed Wolfe type and Mond-Weir type duality results. In [10], Kuk and Tanino derived optimality conditions and duality theorems for non-smooth multiobjective programming problems involving generalized Type I vector valued functions. Gulati and Agarwal [11] discussed sufficiency and duality results for nonsmooth multiobjective problems under ( $F, \alpha, \rho, d$-type I functions. Agarwal et al. [12] established sufficient conditions and duality theorems for nonsmooth multiobjective problems under V-type I functions. Recently, Jayswal et al. [13] obtained some optimality conditions and duality results for nonsmooth multiobjective problems involving generalized $(F, \alpha, \rho, \theta)$ $-d-V$-univexity.

Antczak [14] studied d-invexity is one of the generalization of invex function, which is introduced by [15]. In [14], Antczak established, under weaker assumptions than Ye, the Fritz John type and Karush-Kuhn-Tucker type necessary optimality conditions for weak Pareto optimality and duality results which have been stated in terms of the right differentials of functions involved in the considered multiobjective programming problem. Some authors [16-18] proved that the Karush-KuhnTucker type necessary conditions [14] are sufficient under various generalized d-invex functions. Antczak [19] 
corrrected the Karush-Kuhn-Tucker necessary conditions in [14] and discussed the sufficiency and duality under $d-r$-type I functions. Recently, Silmani and Radjef [20] introduced generalzed $d_{I}$-invexity in which each component of the objective and constraint functions is directionally differentiable in its own direction and established the necessary and sufficient conditions for efficient and properly efficient solutions. The duality results for a Mond-Weir type dual are also derived in [20]. They also observed that the Karush-Kuhn-Tucker sufficient conditions discussed in [16-18] are not applicable. More recently, Agarwal et al. [21] introduced a new class of generalized $d-\rho-(\eta, \theta)$ - type I for a non-smooth multiobjective programming problem and discussed optimality conditions and duality results.

In this paper, we introduce $d_{I}-V$-univexity and generalized $d_{I}-V$-univexity in which each component of the objective and constraint functions of a multiobjective programming problem is semidirectionally differentiable in its own direction $\mathrm{d}_{\mathrm{i}}$. Various Karush-Kuhn-Tucker sufficient optimality conditions for efficient and properly efficient solutions to the problem are established involving new classes of semidirectionally differentiable generalized type I functions. Moreover, usual duality theorems are discussed for a Mond-Weir type dual involving aforesaid assumptions. The results in this paper extend many earlier work appeared in the literature [9,10,12,14-16, 19].

\section{Preliminaries and Definitions}

The following conventions for equalities and inequalities will be used. If $\boldsymbol{x}=\left(x_{1}, \cdots, x_{n}\right), \boldsymbol{y}=\left(y_{1}, \cdots, y_{n}\right) \in \mathbb{R}^{n}$, then $\boldsymbol{x}=\boldsymbol{y} \Leftrightarrow x_{i}=y_{i}, i=1, \cdots, n ; \quad \boldsymbol{x}<\boldsymbol{y} \Leftrightarrow x_{i}<y_{i}, i=1, \cdots, n$; $\boldsymbol{x}<\boldsymbol{y} \Leftrightarrow x_{i}<y_{i}, i=1, \cdots, n ; \quad \boldsymbol{x} \leq \boldsymbol{y} \Leftrightarrow \boldsymbol{x} \leq \boldsymbol{y}$ and $\boldsymbol{x} \neq \boldsymbol{y}$, We also note $\mathbb{R}_{\geq}^{q}$ (resp. $\mathbb{R}_{\geq}^{q}$ or $\mathbb{R}_{\geq}^{q}$ ) the set of vectors $\boldsymbol{y} \in \mathbb{R}^{q}$ with $\boldsymbol{y} \geqq 0$ (resp. $\boldsymbol{y} \geq 0$ or $\boldsymbol{y}>0$ ).

Definition 1 [22]. Let $\mathrm{D}$ be a nonempty subset of $\mathbb{R}^{n}$, $\eta: D \times D \rightarrow \mathbb{R}^{n}$ and let $x_{0}$ be an arbitrary point of $\mathrm{D}$.The set $\mathrm{D}$ is said to be invex at $x_{0}$ with respect to $\eta$, if for each $x \in D$,

$$
x_{0}+\lambda \eta\left(x, x_{0}\right) \in D, \forall \lambda \in[0,1] .
$$

$\mathrm{D}$ is said to be an invex set with respect to $\eta$, if $\mathrm{D}$ is invex at each $x_{0} \in D$ with respect to the same $\eta$.

Definition 2 [23]. Let $D \subseteq \mathbb{R}^{n}$ be an invex set with respect to $\eta: D \times D \rightarrow \mathbb{R}^{n}$.A function $f: D \rightarrow \mathbb{R}$ is called pre-invex on $\mathrm{D}$ with respect to $\eta$, if for all $x, x_{0} \in D$,

$$
\lambda f(x)+(1-\lambda) f\left(x_{0}\right) \geqq f\left(x_{0}+\lambda \eta\left(x, x_{0}\right)\right), \forall \lambda \in[0,1] .
$$

Definition 3 [14]. Let $D \subseteq \mathbb{R}^{n}$ be an invex set with respect to $\eta: D \times D \rightarrow \mathbb{R}^{n}$. A m-dimensional vector valued function $\Psi: D \rightarrow \mathbb{R}^{m}$ is pre-invex with respect to $\eta$, if each of its components is pre-invex on $\mathrm{D}$ with respect to the same function $\eta$.

Definition 4 [7]. Let $D$ be a nonempty open set in $\mathbb{R}^{n}$. A function $f: D \rightarrow \mathbb{R}$ is said to be locally Lipschitz at $x_{0} \in D$, if there exist a neighborhood $v\left(x_{0}\right)$ of $x_{0}$ and a constant $K>0$ such that

$$
|f(y)-f(x)| \geqq K\|y-x\|, \quad \forall x, y \in v\left(x_{0}\right),
$$

where $\|$.$\| denotes the Euclidean norm. We say that f$ is locally Lipschitz on D if its locally Lipschitz at any point of $\mathrm{D}$.

Definition 5 [7]. If $f: D \subseteq \mathbb{R}^{n} \rightarrow R$ is locally Lipschitz at $x_{0} \in D$, the Clarke generalized directional derivative of $f$ at $x_{0}$ in the direction $d \in \mathbb{R}^{n}$, denoted by

$$
f^{0}\left(x_{0} ; d\right)=\lim _{\substack{y \rightarrow x_{0} \\ t \downarrow 0}} \sup \left[\frac{f(y+t d)-f(y)}{t}\right] .
$$

And the usual one-sided directional derivative of $f$ at $x_{0}$ in the direction $d$ is defined by

$$
f^{\prime}\left(x_{0} ; d\right)=\lim _{\lambda \rightarrow 0^{+}} \frac{f\left(x_{0}+\lambda d\right)-f\left(x_{0}\right)}{\lambda},
$$

whenever this limit exists. Obviously,

$$
f^{0}\left(x_{0} ; d\right) \geqq f^{\prime}\left(x_{0} ; d\right) \text {. }
$$

We say that $f$ is directionally differentiable at $x_{0}$ if its directional derivative $f^{\prime}\left(x_{0} ; d\right)$ exists finite for all $d \in \mathbb{R}^{n}$.

Definition 6 [15]. Let $f: D \rightarrow \mathbb{R}^{N}$ be a function defined on a nonempty open set $D \subset \mathbb{R}^{n}$ and directionally differentiable at $x_{0} \in D$. $\mathrm{f}$ is called d-invex at $x_{0}$ on $\mathrm{D}$ with respect to $\eta$, if there exists a vector function $\eta: D \times D \rightarrow \mathbb{R}^{n}$, such that for any $x \in D$,

$$
\begin{aligned}
& |f(y)-f(x)| \geqq K\|y-x\|, \\
& \forall x, y \in v\left(x_{0}\right) f_{i}(x)-f_{i}\left(x_{0}\right) \geqq f_{i}^{\prime}\left(x_{0} ; \eta\left(x, x_{0}\right)\right), \\
& \text { for all } i=1, \cdots, N,
\end{aligned}
$$

where $f_{i}^{\prime}\left(x_{0} ; \eta\left(x, x_{0}\right)\right)$ denotes the directional derivative of $f_{i}$ at $x_{0}$ in the direction

$$
\begin{aligned}
& \eta\left(x, x_{0}\right): f_{i}^{\prime}\left(x_{0} ; \eta\left(x, x_{0}\right)\right) \\
& =\lim _{\lambda \rightarrow 0^{+}} \frac{f_{i}\left(x_{0}+\lambda \eta\left(x, x_{0}\right)\right)-f_{i}\left(x_{0}\right)}{\lambda} .
\end{aligned}
$$

If Inequalities (1) are satisfied at any point $x_{0} \in D$, then $f$ is said to be d-invex on $D$ with respect to $\eta$.

Definition 7 [20]. Let $D$ be a nonempty set in $\mathbb{R}^{n}$ and $\phi: D \times D \rightarrow \mathbb{R}^{n}$ a function.

- We say that $f: D \rightarrow \mathbb{R}$ is a semi-directionally 
differentiable at $x_{0} \in D$,if there exist a nonempty subset $S \subset \mathbb{R}^{n}$ such that $f^{\prime}\left(x_{0} ; d\right)$ exists finite for all $d \in S$

- We say that $f$ is a semi-directionally differentiable at $x_{0} \in D$ in the direction $\phi\left(x, x_{0}\right)$, if its directional derivative $f^{\prime}\left(x_{0} ; \phi\left(x, x_{0}\right)\right)$ exists finite for all $x \in D$.

Definition 8 [20]. Let $f: D \rightarrow \mathbb{R}^{N}$ be a function defined on a nonempty open set $D \subset \mathbb{R}^{n}$ and for all $i=1, \cdots, N, f_{i}$ is semi-directionally differentiable at $x_{0} \in D$ in the direction $\eta_{i}: D \times D \rightarrow \mathbb{R}^{n} . f$ is called $\mathrm{d}_{\mathrm{I}}$-invex at $x_{0}$ on $D$ with respect to $\left(\eta_{i}\right)_{i=\overline{1, N} \text {, }}$ if for any $x \in D$,

$$
f_{i}(x)-f_{i}\left(x_{0}\right) \geqq f_{i}^{\prime}\left(x_{0} ; \eta_{i}\left(x, x_{0}\right)\right) \text {, for all } i, 2, \cdots, N,
$$

where $f_{i}^{\prime}\left(x_{0} ; \eta_{i}\left(x, x_{0}\right)\right)$ denotes the directional derivative of $f_{i}$ at $x_{0}$ in the direction

$$
\begin{aligned}
& \eta_{i}\left(x, x_{0}\right): f_{i}^{\prime}\left(x_{0} ; \eta_{i}\left(x, x_{0}\right)\right) \\
& =\lim _{\lambda \rightarrow 0^{+}} \frac{f_{i}\left(x_{0}+\lambda \eta_{i}\left(x, x_{0}\right)\right)-f_{i}\left(x_{0}\right)}{\lambda} .
\end{aligned}
$$

If Inequalities (2) are satisfied at any point $x_{0} \in D$, then $f$ is said to be $d_{I}$-invex on $D$ with respect to $\left(\eta_{i}\right)_{i=\overline{1, N},}$

Consider the following multiobjective programming problem

$(M P)$ Minimize $f(x)=\left(f_{1}(x), f_{2}(x), \cdots, f_{N}(x)\right)$

Subject to $g(x) \leqq 0$,

$$
x \in D \text {, }
$$

where $f: D \rightarrow \mathbb{R}^{N}, g: D \rightarrow \mathbb{R}^{k}$, D is a nonempty open subset of $\mathbb{R}^{n}$. Let $X=\{x \in D: g(x) \leqq 0\}$ be the set of feasible solutions of (MP). For $x_{0} \in D$, we denote by $J\left(x_{0}\right)$ the set $\left\{j \in\{1,2, \cdots, k\}: g_{j}\left(x_{0}\right)=0\right\}, J=\left|J\left(x_{0}\right)\right|$ and by $\tilde{J}\left(x_{0}\right)\left(\operatorname{resp} . \bar{J}\left(x_{0}\right)\right)$ the set $\left\{j \in\{1,2, \cdots, k\}: g_{j}\left(x_{0}\right)<0\right.$ (resp. $\left.\left.g_{j}\left(x_{0}\right)>0\right)\right\}$. we have $J\left(x_{0}\right) \cup \tilde{J}\left(x_{0}\right) \cup \bar{J}\left(x_{0}\right)=\{1,2, \cdots, k\}$ and if $x_{0} \in X, \bar{J}\left(x_{0}\right)=\varnothing$.

We recall some optimality concepts, the most often studied in the literature, for the problem (MP).

Definition 9. A point $x_{0} \in X$ is said to be a local weakly efficient solution of the problem (MP), if there exists a neighborhood $N\left(x_{0}\right)$ around $x_{0}$ such that

$$
f(x) \nless f\left(x_{0}\right) \text { for all } x \in N\left(x_{0}\right) \cap X
$$

Definition 10. A Point $x_{0} \in X$ is said to be a weakly efficient (an efficient) solution of the problem (MP), if there exists no $x \in X$ such that

$$
f(x)<f\left(x_{0}\right)\left(f(x) \leq f\left(x_{0}\right)\right) .
$$

Definition 11. An efficient solution $x_{0} \in X$ of (MP) is said to be properly efficient, if there exists a positive real number $M$ such that inequality

$$
f_{i}\left(x_{0}\right)-f_{i}(x) \leqq M\left[f_{j}(x)-f_{j}\left(x_{0}\right)\right]
$$

is verified for all $i \in\{1, \cdots, N\}$ and $x \in X$ such that $f_{i}(x)<f_{i}\left(x_{0}\right)$, and for a certain $j \in\{1, \cdots, N\}$ such that $f_{j}(x)>f_{j}\left(x_{0}\right)$.

Following Jeyakumar and Mond [24], Kaul et al. [8] and Slimani and Radjef [20], we give the following definitions.

Definition 12. $(f, g)$ is $d_{I}-V$-univex type $\mathrm{I}$ at $x_{0} \in D$ if there exist positive real valued functions $\alpha_{i}$ and $\beta_{j}$ defined on $X \times D$, nonnegative functions $b_{0}$ and $b_{1}$, also defined on $X \times D, \phi_{0}: R \rightarrow R, \phi_{1}: R \rightarrow$ $R ; \eta_{i}: X \times D \rightarrow R^{n}$, and $\theta_{j}: X \times D \rightarrow R^{n}$ such that $b_{0}\left(x, x_{0}\right) \phi_{0}\left[f_{i}(x)-f_{i}\left(x_{0}\right)\right] \geqq \alpha_{i}\left(x, x_{0}\right) f_{i}^{\prime}\left(x_{0} ; \eta_{i}\left(x, x_{0}\right)\right)$

and

$$
-b_{1}\left(x_{1}, x_{0}\right) \phi_{1}\left[g_{j}\left(x_{0}\right)\right] \geqq \beta_{j}\left(x, x_{0}\right) g_{j}^{\prime}\left(x_{0} ; \theta_{j}\left(x, x_{0}\right)\right)
$$

for every $\quad x \in X$ and for all $i=1,2, \cdots, N$ and $j=1,2, \cdots, k$.

If the inequality in (3) is strict (whenever $x \neq x_{0}$ ), we say that (MP) is of semistrictly $d_{I}-V$-univex type I at $x_{0}$ with respect to $\left(\eta_{i}\right)_{i=\overline{1, N}}$ and $\left(\theta_{j}\right)_{j=\overline{1, k}}$.

Definition 13. $(f, g)$ is quasi- $d_{I}-V$-univex type I at $x_{0} \in D$ if there exist positive real valued functions $\alpha$ and $\beta_{j}$, defined on $X \times D$, nonnegative functions $b_{0}$ and $b_{1}$, also defined on $X \times D, \varphi_{0}: R \rightarrow R$,

$\varphi_{1}: R \rightarrow R$ and $(N+k)$ dimensional vector functions $\eta_{i}: X \times D \rightarrow R^{n}, i=\overline{1, N}$ and $\theta_{j}: X \times D \rightarrow R^{n}, j=\overline{1, k}$ such that for some vectors $\lambda \in R_{\geqq}^{N}$ and $\mu \in R_{\geqq}^{k}$ :

$$
\begin{aligned}
& b_{0}\left(x, x_{0}\right) \phi_{0}\left[\sum_{i=1}^{N} \lambda_{i} \alpha_{i}\left(x, x_{0}\right)\left(f_{i}(x)-f_{i}\left(x_{0}\right)\right)\right] \\
& \leqq 0 \Rightarrow \sum_{i=1}^{N} \lambda_{i} f_{i}^{\prime}\left(x_{0} ; \eta_{i}\left(x, x_{0}\right)\right) \leqq 0 \quad \forall x \in X
\end{aligned}
$$

and

$$
\begin{aligned}
& b_{1}\left(x, x_{0}\right) \phi_{1}\left[\sum_{j=1}^{k} \mu_{j} \beta_{j}\left(x, x_{0}\right) g_{j}\left(x_{0}\right)\right] \geqq 0 \\
& \Rightarrow \sum_{j=1}^{k} \mu_{j} g_{j}^{\prime}\left(x_{0} ; \theta_{j}\left(x, x_{0}\right)\right) \geqq 0 \quad \forall x \in X .
\end{aligned}
$$

If the second inequality in (5) is strict $\left(x \neq x_{0}\right)$, we say that (MP) is of semi-strictly quasi $d_{I}$-V-univex type I at $X$ with respect to $\left(\eta_{i}\right)_{i=\overline{1, N}}$ and $\left(\theta_{j}\right)_{j=\overline{1, k}}$. 
Definition 14. $(f, g)$ is pseudo- $d_{I}-V$-univex type I at $x_{0} \in D$ if there exist positive real valued functions $\alpha_{i}$ and $\beta_{j}$, defined on $X \times D$, nonnegative functions $b_{0}$ and $b_{1}$, also defined on $X \times D, \phi_{0}: R \rightarrow R$, $\phi_{1}: R \rightarrow R$ and $(N+k)$ dimensions vector functions $\eta_{i}: X \times D \rightarrow R^{n}, i=\overline{1, N}$ and $\theta_{j}: X \times D \rightarrow R^{n}, j=\overline{1, k}$ such that for some vectors $\lambda \in R_{\geq}^{N}$ and $\mu \in R_{\geq}^{k}$ :

$$
\begin{aligned}
& \sum_{i=1}^{N} \lambda_{i} f_{i}^{\prime}\left(x_{0} ; \eta_{i}\left(x, x_{0}\right)\right) \geqq 0 \Rightarrow \\
& b_{0}\left(x, x_{0}\right) \phi_{0}\left[\sum_{i=1}^{N} \lambda_{i} \alpha_{i}\left(x, x_{0}\right)\left(f_{i}(x)-f_{i}\left(x_{0}\right)\right)\right] \leqq 0 \quad \forall x \in X
\end{aligned}
$$

and

$$
\begin{aligned}
& \sum_{j=1}^{k} \mu_{j} g_{j}^{\prime}\left(x_{0} ; \theta_{j}\left(x, x_{0}\right)\right) \geqq 0 \Rightarrow \\
& b_{1}\left(x, x_{0}\right) \phi_{1}\left[\sum_{j=1}^{k} \mu_{j} \beta_{j}\left(x, x_{0}\right) g_{j}\left(x_{0}\right)\right] \leqq 0 \quad \forall x \in X .
\end{aligned}
$$

Definition 15. $(f, g)$ is quasi pseudo- $d_{I}-V$-univex type I at $x_{0} \in D$ if there exist positive real valued functions $\alpha_{i}$ and $\beta_{i}$, defined on $X \times D$, nonnegative functions $b_{0}$ and $b_{1}$, also defined on $X \times D, \phi_{0}: R \rightarrow R$, $\phi_{1}: R \rightarrow R$ and $(N+k)$ dimensions vector functions $\eta_{i}: X \times D \rightarrow R^{n}, i=\overline{1, N}$ and $\theta_{j}: X \times D \rightarrow R^{n}, j=\overline{1, k}$ such that the relation (5) and (8) are satisfied. If the second inequality in (8) is strict $\left(x \neq x_{0}\right.$, we say that $(V P)$ is of quasi strictly-pseudo $\mathrm{d}_{\mathrm{I}}-\mathrm{V}$-type $I$ at $x_{0}$ with respect to $\left(\eta_{i}\right)_{i=\overline{1, N}}$ and $\left(\theta_{j}\right)_{j=\overline{1, k}}$.

Definition 16. $(f, g)$ is pseudoquasi $-\mathrm{d}_{\mathrm{I}}-\mathrm{V}$-univex type I at $x_{0} \in D$ if there exist positive real valued functions $\alpha_{i}$ and $\beta_{j}$, defined on $X \times D$, nonnegative functions $b_{0}$ and $b_{1}$, also defined on $X \times D$, $\phi_{0}: R \rightarrow R, \phi_{1}: R \rightarrow R$ and $(N+k)$ dimensions vector functions $\eta_{i}: X \times D \rightarrow R^{n}, i=\overline{1, N}$ and $\theta_{j}: X \times D \rightarrow R^{n}, j=\overline{1, k}$, such that $\mu \in R_{\geqq}^{k}$ the relations (7) and (6) are satisfied. If the second inequality in (7) is strict $\left(x \neq x_{0}\right)$, we say that (MP) is of strictlypseudo quasi $d_{I}-\mathrm{V}$-type $\mathrm{I}$ at $x_{0}$ with respect to $\left(\eta_{i}\right)_{i=\overline{1, N}}$ and $\left(\theta_{j}\right)_{j=\overline{1, k}}$.

\section{Optimality Conditions}

In this section, we discuss some sufficient conditions for a point to be an efficient or properly efficient for (MP) under generalized $d_{I}-V$ - univex type I assumptions.

Theorem 3.1. Let $x_{0}$ be a feasible solution for (MP) and suppose that there exist $(\boldsymbol{N}+\boldsymbol{J})$ vector functions $\eta_{i}: X \times X \rightarrow R^{n}, i=\overline{1, N}, \quad \theta_{j}: X \times X \rightarrow R^{n}, j \in J\left(x_{0}\right)$ and scalars $\bar{\lambda}_{i} \geqq 0, i=\overline{1, N}, \sum_{i=1}^{N} \bar{\lambda}_{i}=1 ; \quad \bar{\mu}_{j} \geqq 0$, $j \in J\left(x_{0}\right)$ such that

$$
\begin{aligned}
& \sum_{i=1}^{N} \bar{\lambda}_{i} f_{i}^{\prime}\left(x_{0} ; \eta_{i}\left(x, x_{0}\right)\right)+\sum_{j \in J\left(x_{0}\right)} \bar{\mu}_{j} g_{j}^{\prime}\left(x_{0} ; \theta_{j}\left(x, x_{0}\right)\right) \geqq 0, \\
& \forall x \in X,
\end{aligned}
$$

Further, assume that one of the following conditions is satisfied:

a) i) $(f, g)$ is quasi strictly-pseudo $d_{I}-V$-univex type I at $x_{0}$ with respect to $\left(\eta_{i}\right)_{i=1, N},\left(\theta_{j}\right)_{j \in J\left(x_{0}\right)}, \bar{\lambda}, \bar{\mu}$ and for some positive functions $\alpha_{i}, i=\overline{1, N}, \quad \beta_{j}$, $j \in J\left(x_{0}\right)$,

ii) for any $u \in R, u \leqq 0 \Rightarrow \varphi_{0}(u) \leqq 0 ; \quad \varphi_{1}(u)<0$ $\Rightarrow u<0 ; \quad b_{0}\left(x, x_{0}\right)>0, \quad b_{1}\left(x, x_{0}\right)>0$;

b) i) $(f, g)$ is strictly-pseudo $d_{I}-V$-univex type I at $x_{0}$ with respect to $\left(\eta_{i}\right)_{i=\overline{1, N}},\left(\theta_{j}\right)_{j \in J\left(x_{0}\right)}, \bar{\lambda}, \bar{\mu}$ and for some positive functions $\alpha_{i}, i=\overline{1, N}, \beta_{j}$, $j \in J\left(x_{0}\right)$,

ii) for any $u \in R, \varphi_{0}(u)>0 \Rightarrow u>0$; $u \geqq 0 \Rightarrow \varphi_{1}(u) \geqq 0, b_{0}\left(x, x_{0}\right)>0, \quad b_{1}\left(x, x_{0}\right) \geqq 0$. Then $x_{0}$ is an efficient solution for (MP).

Proof: Condition a). Suppose that $x_{0}$ is not an efficient solution of (MP). Then there exists an $x \in X$ such that

$$
f(x) \leq f\left(x_{0}\right),
$$

which implies that

$$
\sum_{i=1}^{N} \bar{\lambda}_{i} \alpha_{i}\left(x, x_{0}\right)\left[f_{i}(x)-f_{i}\left(x_{0}\right)\right] \leqq 0 .
$$

Since $b_{0}\left(x, x_{0}\right)>0 ; u \geqq 0 \Rightarrow \varphi_{0}(u) \geqq 0$, the above inequality gives

$$
b_{0}\left(x, x_{0}\right) \phi_{0}\left[\sum_{i=1}^{N} \bar{\lambda}_{i} \alpha_{i}\left(x, x_{0}\right)\left[f_{i}(x)-f_{i}\left(x_{0}\right)\right]\right] \leqq 0 .
$$
have

From the above inequality and Hypothesis i) of a), we

$$
\sum_{i=1}^{N} \bar{\lambda}_{i} f_{i}^{\prime}\left(x_{0} ; \eta_{i}\left(x, x_{0}\right)\right) \leqq 0 .
$$

By using the Inequality (9) we deduce that

$$
\sum_{j \in J\left(x_{0}\right)} \bar{\mu}_{j} g_{j}^{\prime}\left(x_{0} ; \theta_{j}\left(x, x_{0}\right)\right) \geqq 0,
$$

which implies from the condition part ii) of a) that

$$
b_{1}\left(x, x_{0}\right) \phi_{1}\left[\sum_{j \in J\left(x_{0}\right)} \bar{\mu}_{j} \beta_{j}\left(x, x_{0}\right) g_{j}\left(x_{0}\right)\right]<0 .
$$

Since $b_{1}\left(x, x_{0}\right)>0 ; \phi_{1}(u)<0 \Rightarrow u<0$, we get 


$$
\sum_{j \in J\left(x_{0}\right)} \bar{\mu}_{j} \beta_{j}\left(x, x_{0}\right) g_{j}\left(x_{0}\right)<0 .
$$

As $\bar{\lambda} \geqq 0$ and $g_{j}\left(x_{0}\right)=0 ; \forall j \in J\left(x_{0}\right)$, it follows that $\bar{\lambda}_{j} g_{j}\left(x_{0}\right)=0, \forall j \in J\left(x_{0}\right)$, which implies that

$$
\sum_{j \in J\left(x_{0}\right)} \bar{\mu}_{j} \beta_{j}\left(x, x_{0}\right) g_{j}\left(x_{0}\right)=0 .
$$

The above equation contradicts Inequality (11) and hence the conclusion of the theorem follows:

Condition b): Since $g_{j}\left(x_{0}\right)=0, \bar{\mu}_{j} \geqq 0, \forall j \in J\left(x_{0}\right)$, and $\beta_{j}\left(x, x_{0}\right)>0, j \in J\left(x_{0}\right)$, we obtain

$$
\sum_{j \in J\left(x_{0}\right)} \bar{\mu}_{j} \beta_{j}\left(x, x_{0}\right) g_{j}\left(x_{0}\right)=0, \quad \forall x \in X .
$$

By Hypothesis ii) of b), we get

$$
b_{1}\left(x, x_{0}\right) \phi_{1}\left[\sum_{j \in J\left(x_{0}\right)} \bar{\mu}_{j} \beta_{j}\left(x, x_{0}\right)\right] \geqq 0 .
$$

From the above inequality and the Hypothesis i) of b)( in view of reverse implication in (8), if follows that

$$
\sum_{j \in J\left(x_{0}\right)} \bar{\mu}_{j} g_{j}^{\prime}\left(x_{0} ; \theta_{j}\left(x, x_{0}\right)\right)<0, \quad \forall x \in X \backslash\left\{x_{0}\right\} .
$$

By using Inequality (9), we deduce that

$$
\sum_{i=1}^{N} \bar{\lambda}_{i} f_{i}^{\prime}\left(x_{0} ; \eta_{i}\left(x, x_{0}\right)\right)>0, \quad \forall x \in X \backslash\left\{x_{0}\right\},
$$

which by virtue of relation (7) implies that

$$
\begin{aligned}
& b_{0}\left(x, x_{0}\right) \phi_{0}\left[\sum_{i=1}^{N} \bar{\lambda}_{i} \alpha_{i}\left(x, x_{0}\right)\left(f_{i}(x)-f_{i}\left(x_{0}\right)\right)\right]>0, \\
& \forall x \in X \backslash\left\{x_{0}\right\} .
\end{aligned}
$$

The above inequality along with Hypothesis ii) of b) gives

$$
\sum_{i=1}^{N} \bar{\lambda}_{i} \alpha_{i}\left(x, x_{0}\right)\left(f_{i}(x)-f_{i}\left(x_{0}\right)\right)>0, \forall x \in X \backslash\left\{x_{0}\right\} .
$$

Since (10) and (13) contradicts each other, and hence the conclusion follows:

Theorem 3.2. Let $x_{0}$ be a feasible solution for (MP) and suppose that there exist $(N+J)$ vector functions $\eta_{i}: X \times X \rightarrow R^{n}, i=\overline{1, N}, \theta_{j}: X \times X \rightarrow R^{n}, j \in J\left(x_{0}\right)$ and scalars $\bar{\lambda}_{i} \geqq 0, i=\overline{1, N}, \sum_{i=1}^{N} \bar{\lambda}_{i}=1, \bar{\mu}_{j} \geqq 0, j \in J\left(x_{0}\right)$ such that Inequality (9) of Theorem 3.1 is satisfied.

Moreover, assume that one of the following conditions is satisfied.

a) i) $(f, g)$ is pseudo quasi $d_{I}-V$-univex type $I$ at $x_{0}$ with respect to $\left(\eta_{i}\right)_{i=\overline{1, N}},\left(\theta_{j}\right)_{j \in J\left(x_{0}\right)}, \bar{\lambda}, \bar{\mu}$ and for some positive functions $\alpha_{i}, i=\overline{1, N}$ and $\beta_{j}, j \in J\left(x_{0}\right)$,

ii) for any $u \in R$,

$$
\begin{gathered}
u \geqq 0 \Rightarrow \phi_{1}(u) \geqq 0, \phi_{0}(u) \geqq 0 \Rightarrow u \geqq 0, \\
b_{0}\left(x, x_{0}\right)>0, b_{1}\left(x, x_{0}\right) \geqq 0 ;
\end{gathered}
$$

b) i) $(f, g)$ is strictly pseudo $d_{I}-V$-univex type I at $x_{0}$ with respect to $\left(\eta_{i}\right)_{i=\overline{1, N}},\left(\theta_{j}\right)_{j \in J\left(x_{0}\right)}, \bar{\lambda}, \bar{\mu}$ and for positive functions $\alpha_{i}=\overline{1, N}$ and $\beta_{j}, j \in J\left(x_{0}\right)$,

ii) for any $u \in R$

$$
\begin{aligned}
& u \leqq 0 \Rightarrow \phi_{0}(u) \leqq 0 ; u \geqq 0 \Rightarrow \phi_{1}(u) \geqq 0 ; \\
& b_{0}\left(x, x_{0}\right)>0, b_{1}\left(x, x_{0}\right) \geqq 0 .
\end{aligned}
$$

Then $x_{0}$ is an efficient solution for (MP). Further Suppose that these exist positive real numbers $n_{i}, m_{i}$ such that $n_{i}<\alpha_{i}\left(x, x_{0}\right)<m_{i}, i=\overline{1, N}$ for all feasible $\mathrm{X}$.Then $\mathrm{X}_{0}$ is a properly efficient solution for (MP)

Proof: Condition a). Suppose that $x_{0}$ is not an efficient solution of (MP). Then there exists an $x \in X$ such that $f(x) \leq f\left(x_{0}\right)$ which implies that

$$
\sum_{i=1}^{N} \bar{\lambda}_{i} \alpha_{i}\left(x, x_{0}\right)\left(f_{i}(x)-f_{i}\left(x_{0}\right)\right)<0 .
$$

Since $g_{j}\left(x_{0}\right)=0, \bar{\mu}_{j} \geqq 0$ and

$$
\beta_{j}\left(x, x_{0}\right)>0, \forall j \in J\left(x_{0}\right)
$$

we obtain

$$
\sum_{j \in J\left(x_{0}\right)} \mu_{j} \beta_{j}\left(x, x_{0}\right) g_{j}\left(x_{0}\right)=0 .
$$

From the above inequality and Hypothesis ii) of a), we have

$$
b_{1}\left(x, x_{0}\right) \phi_{1}\left[\sum_{j \in J\left(x_{0}\right)} \bar{\mu}_{j} \beta_{j}\left(x, x_{0}\right) g_{j}\left(x_{0}\right)\right] \geqq 0 .
$$

Using Hypothesis i) of a), we deduce that

$$
\sum_{j \in J\left(x_{0}\right)} \bar{\mu}_{j} \beta_{j}\left(x, x_{0}\right) g_{j}^{\prime}\left(x_{0} ; \theta_{j}\left(x, x_{0}\right)\right) \leqq 0 .
$$

The Inequalities (9) and (14) yield that

$$
\sum_{i=1}^{N} \bar{\lambda}_{i} f_{i}^{\prime}\left(x_{0} ; \eta_{i}\left(x, x_{0}\right)\right) \geqq 0,
$$

which by Hypothesis i) of a), we obtain

$$
b_{0}\left(x, x_{0}\right) \phi_{0}\left[\sum_{i=1}^{N} \bar{\lambda}_{i} \alpha_{i}\left(x, x_{0}\right)\left(f_{i}(x)-f_{i}\left(x_{0}\right)\right)\right] \geqq 0,
$$

The Inequality (16) and Hypothesis ii) of a) give

$$
\sum_{i=1}^{N} \bar{\lambda}_{i} \alpha_{i}\left(x, x_{0}\right)\left(f_{i}(x)-f_{i}\left(x_{0}\right)\right) \geqq 0 .
$$

Since (14) and (17) contradict each other, we conclude 
that $x_{0}$ is not an efficient solution of (MP). The properly efficient solution follows as in Hanson et al. [25]. For the proof of part b), we proceed as in part b) of Theorem 3.1, we get Inequality (17). Thus complete the proof.

\section{Mond-Weir Type Duality}

Consider the following multiobjective dual to problem (MP)

(MD) Maximize $f(y)=\left(f_{1}(y), f_{2}(y), \cdots, f_{N}(y)\right)$ subject to

$$
\begin{gathered}
\sum_{i=1}^{N} \lambda_{i} f_{i}^{\prime}\left(y ; \eta_{i}(x, y)\right)+\sum_{j=1}^{k} \mu_{j} g_{j}^{\prime}\left(y ; \theta_{j}(x, y)\right) \geqq 0, \forall x \in X \\
\mu_{j} g_{j}(y) \geqq 0, j=1,2, \cdots, k, y \in D, \lambda \in R_{\geqq}^{N}, \mu \in R_{\geqq}^{k} \\
\eta_{i}: X \times D \rightarrow R^{n}, \quad \forall i=1,2, \cdots, N, \\
\theta_{j}: X \times D \rightarrow R^{n}, j=1,2, \cdots, k .
\end{gathered}
$$

Let $Y$ be the set of feasible solutions of problem (MD); that is,

$$
\begin{aligned}
& Y=\left\{\left(y, \lambda, \mu,\left(\eta_{i}\right)_{i},\left(\theta_{j}\right)_{j}\right):\right. \\
& \sum_{i=1}^{N} \lambda_{i} f_{i}^{\prime}\left(y ; \eta_{2}(x, y)\right)+\sum_{j=1}^{k} \mu_{j} g_{j}^{\prime}\left(y ; \theta_{j}(x, y)\right) \geqq 0, \\
& \mu_{j} g_{j}(y) \geqq 0, \quad \forall x \in X ; y \in D, \lambda \in R_{\geqq}^{N}, \mu \in R_{\geqq}^{k} ; \\
& \eta_{i}: X \times D \rightarrow R^{n} \quad \forall i=1,2, \cdots, N ; \\
& \left.\theta_{j}: X \times D \rightarrow R^{n}, \forall j=1,2, \cdots, k\right\} .
\end{aligned}
$$

We denote by $P_{r D} Y$, the projection of set $Y$ on $D$.

Theorem 4.1. (Weak Duality). Let $x$ and $\left(y, \lambda, \mu,\left(\eta_{i}\right)_{i=\overline{1, N}},\left(\theta_{j}\right)_{j=\overline{1, k}}\right)$ be feasible solution for (MP) and (MD) respectively. Moreover, assume that one of the following conditions is satisfied:

a) i) $(f, g)$ is pseudo quasi $d_{I}-\mathrm{V}$-univex type $\mathrm{I}$ at $y$ with respect to $\lambda>0, \mu,\left(\eta_{i}\right)_{i=\overline{1, N}},\left(\theta_{j}\right)_{j=1, k}$ and for some positive functions $\alpha_{i}, \beta_{j}$ for $i=1,2, \cdots, N$ and $j=1,2, \cdots, k$,

ii) for any $u \in R$

$$
\begin{aligned}
& \phi_{0}(u) \geqq 0 \Rightarrow u \geqq 0 ; u \geqq 0 \Rightarrow \phi_{1}(u) \geqq 0 ; \\
& b_{0}(x, y)>0, b_{1}(x, y) \geqq 0
\end{aligned}
$$

b) i) $(f, g)$ is strictly-pseudo quasi $d_{I}$-V-univex type $I$ at $y$ with respect to $\lambda, \mu,\left(\eta_{i}\right)_{i=\overline{1, N}},\left(\theta_{j}\right)_{j=\overline{1, k}}$ and for some positive function $\alpha_{i}, \beta_{j}$ for $i=1,2, \cdots, N$ and $j=1,2, \cdots, k$,

ii) for any $u \in R$,

$$
\begin{aligned}
& \phi_{0}(u) \geqq 0 \Rightarrow u>0 ; u \geqq 0 \Rightarrow \phi_{1}(u) \geqq 0 ; \\
& b_{1}(x, y) \geqq 0, b_{0}(x, y)>0 ;
\end{aligned}
$$

c) i) $(f, g)$ is quasi strictly-pseudo $d_{I}-V$-univex type I at $y$ with respect to $\lambda, \mu,\left(\eta_{i}\right)_{i=\overline{1, N}},\left(\theta_{j}\right)_{j=\overline{1, k}}$ and for some positive functions $\alpha_{i}, \beta_{j}$ for $i=$ $1,2, \cdots, N$ and $j=1,2, \cdots, k$,

ii) for any $u \in R$,

$$
\begin{aligned}
& \phi_{0}(u)>0 \Rightarrow u>0 ; u>0 \Rightarrow \phi_{1}(u)>0 ; \\
& b_{0}(x, y)>0, b_{1}(x, y)>0 .
\end{aligned}
$$

Then $f(x) \not \leq f(y)$.

Proof: Since

$$
\begin{aligned}
& \mu_{j} g_{j}(y) \geqq 0, j=1,2, \cdots, k, \\
& u \geqq 0 \Rightarrow \phi_{1}(u) \geqq 0, b_{1}(x, y)>0, \\
& \text { and } \beta_{j}(x, y)>0, j=1,2, \cdots, k
\end{aligned}
$$

we have

$$
b_{1}(x, y) \phi_{1}\left[\sum_{j=1}^{k} \mu_{j} \beta_{j}(x, y) g_{j}(y)\right] \geqq 0 .
$$
that

By Condition a) (in view of definition 16), it follows

$$
\sum_{j=1}^{k} \mu_{j} \beta_{j}(x, y) g_{j}\left(y ; \theta_{j}(x, y)\right) \leqq 0 .
$$

Since $\left(y, \lambda, \mu,\left(\eta_{i}\right)_{i=\overline{1, N}},\left(\theta_{j}\right)_{j=\overline{1, k}}\right)$ is a feasible solution for (MD), the first dual constraint with (18) implies that

$$
\sum_{i=1}^{N} \lambda_{i} f_{i}^{\prime}\left(y ; \eta_{i}(x, y)\right) \geqq 0 .
$$

From (19) and Hypothesis i) of a), we obtain

$$
b_{0}(x, y) \phi_{0}\left[\sum_{i=1}^{N} \lambda_{i} \alpha_{i}(x, y)\left(f_{i}(x)-f_{i}(y)\right)\right] \geqq 0 \text {. }
$$

Condition ii) of a) and Inequality (20) give

$$
\sum_{i=1}^{N} \lambda_{i} \alpha_{i}(x, y)\left(f_{i}(x)-f_{i}(y)\right) \geqq 0 .
$$

Assume that $f(x) \leq f(y)$. Since $\alpha_{i}>0, i=1,2, \cdots, N$ and $\lambda>0$, we obtain

$$
\sum_{i=1}^{N} \lambda_{i} \alpha_{i}(x, y)\left(f_{i}(x)-f_{i}(y)\right)<0
$$

which contradicts (21), Therefore, the conclusion follows:

The proof of part b) and c) are very similar to proof of part a), except that: for part b), the Inequality (21) becomes strict $(>)$ and Inequality (22) becomes non strict $(\leqq)$. For part c), the Inequality (18) becomes strict $(<)$, 
it follows that the Inequalities (20) and (21) become strict $(>)$. Since $\lambda \geqq 0$, then the Inequality (22) becomes non strict $(\leqq)$. In this cases, the Inequalities (21) and (22) contradicts each other always.

Remark 1: If we omit the assumption $\lambda>0$ in the condition i) of a) or the word "strictly" in the condition b), we obtain, for this part of theorem, $f(x) \not \leq f(y)$.

Theorem 4.2. (Weak Duality). Let $x$ and $\left(y, \lambda, \mu,\left(\eta_{i}\right)_{i=\overline{1, N}},\left(\theta_{j}\right)_{j=\overline{1, K}}\right)$ be feasible solutions for (MP) and (MD) respectively, Assume that

1) $(f, g)$ is semi-strictly $d_{I}-V$-univex type $I$ at $y$ with respect to $\lambda>0, \mu,\left(\eta_{i}\right)_{i=1, \bar{N}},\left(\theta_{j}\right)_{j=1, \bar{k}}$ and for some positive functions $\left(\alpha_{i}^{\star}\right)_{i=\overline{1, N}},\left(\beta_{j}^{\star}\right)_{j=\overline{1, k}}$,

2) for any $u \in R$,

$$
\begin{aligned}
& \phi_{0}(u)>0 \Rightarrow a>0,(u) \geqq 0 \Rightarrow \phi_{1}(u) \geqq 0, \\
& b_{0}(x, y)>0, b_{1}(x, y) \geqq 0 .
\end{aligned}
$$

Then $f(x) \not \leq f(y)$.

Proof: Since $\mu_{j} g_{j}(y) \geqq 0, j=1,2, \cdots, k$, which implies that

$$
\sum_{j=1}^{k} \mu_{j} \beta_{j}^{\star}(x, y) g_{j}(y) \geqq 0 .
$$

By (23) and Hypothesis i) (with $\left.b_{1}(x, y) / \beta_{j}(x, y)\right)$ in Definition 12 replaced by

$$
\beta_{j}^{\star}(x, y) \text { it follows that }
$$

$$
\sum_{j=1}^{k} \mu_{j} g_{j}^{\prime}\left(y, \theta_{j}(x, y)\right) \leqq 0 .
$$

The first dual constraint and (24) give

$$
\sum_{i=1}^{N} \lambda_{i} f_{i}^{\prime}\left(y, \eta_{i}(x, y)\right) \geqq 0 .
$$

Dividing both sides of (3) by $\alpha_{i}(x, y)$ and taking $x \neq y$, by Hypothesis i), we get

$$
\begin{aligned}
& b_{0}(x, y) \frac{1}{\alpha_{i}(x, y)} \phi_{0}\left[f_{i}(x)-f_{i}(y)\right]>f_{i}^{\prime}\left(y, \eta_{i}(x, y)\right), \\
& i=1,2, \cdots, N .
\end{aligned}
$$
get

On Multiplying by $\lambda_{i}$ and taking $\alpha_{i}^{\star}=\frac{1}{\alpha_{i}(x, y)}$, we

$$
\begin{aligned}
& b_{0}(x, y) \lambda_{i} \alpha_{i}^{\star} \phi_{0}\left[f_{i}(x)-f_{i}(y)\right]>\lambda_{i} f_{i}^{\prime}\left(y, \eta_{i}(x, y)\right), \\
& i=1,2, \cdots, N
\end{aligned}
$$

Adding with respect to $i$, and applying (25) and Hypothesis ii), we have

$$
\sum_{i=1}^{N} \lambda_{i} \alpha_{i}^{\star}(x, y)\left(f_{i}(x)-f_{i}(y)\right)>0 .
$$

Assume that $f(x) \leq f(y)$. Since $\alpha_{i}^{\star}>0$ and $\lambda>0$, we have

$$
\sum_{i=1}^{N} \lambda_{i} \alpha_{i}^{\star}(x, y)\left(f_{i}(x)-f_{i}(y)\right)<0,
$$

which contradicts (26).

Theorem 4.3. (Strong Duality ).Let $x_{0}$ be a weakly efficient solution for (MP). Assume that the function $g$ satisfies the $d_{I}$-constraint qualification at $x_{0}$ with respect to $\left(\theta_{j}\right)_{j=\overline{1, k}}$. Then there exist $\lambda \in R_{>}^{N}$ and $\mu \in R_{>}^{K}$ such that $\left(x_{0}, \lambda, \mu, \eta_{i}\right)_{i=\overline{1, N}},\left(\theta_{j}\right)_{j=1, \bar{k}} \in Y$ and objective functions of (MP) and (MD) have the same values at $x_{0}$ and $\left(x_{0}, \lambda, \mu,\left(\eta_{i}\right)_{i=\overline{1, N}},\left(\theta_{j}\right)_{j=1, \bar{k}}\right)$, respectively. If, further, the weak duality between (MP) and (MD) in theorem holds with the condition a) without $\lambda>0$ (resp. with the condition b) or c)), then $\left(x_{0}, \lambda, \mu,\left(\eta_{i}\right)_{i=1, N},\left(\theta_{j}\right)_{j=\overline{1, k}}\right) \in Y$ is a weakly efficient (resp. an efficient) solutions of (MD).

Proof. By the Theorem 31 [20], there exists $\mu \in \mathbb{R}_{\geq}^{k}$ and $\lambda \in \mathbb{R}_{>}^{J\left(x_{0}\right) \mid}$ such that

$$
\sum_{i=1}^{N} \lambda_{i} f_{i}^{\prime}\left(x_{0} ; \eta_{i}\left(x, x_{0}\right)\right)+\sum_{j=1}^{k} \mu_{j} g_{j}^{\prime}\left(x_{0} ; \theta_{j}\left(x, x_{0}\right)\right) \geqq 0,
$$

$\forall x \in X$.

It follows that $\left(x_{0}, \mu, \lambda,\left(\eta_{i}\right)_{i=\overline{1, N}},\left(\theta_{j}\right)_{j=\overline{1, k}}\right) \in Y$. Trivially, the objective function values of (MP) and (MD) are equal.

Suppose that $\left(x_{0}, \lambda, \mu,\left(\eta_{i}\right)_{i=\overline{1, N}},\left(\theta_{j}\right)_{j=\overline{1, k}}\right) \in Y$ is not a weakly efficient solution of (MD). Then there exists $\left(y^{\star}, \lambda^{\star}, \mu^{\star},\left(\eta_{i}^{\star}\right)_{i=\overline{1, N}},\left(\theta_{j}^{\star}\right)_{j=\overline{1, k}}\right) \in Y$ such that $f\left(x_{0}\right)<f\left(y^{\star}\right)$ which violates the weak duality theorem. Hence $\left(x_{0}, \lambda, \mu,\left(\eta_{i}\right)_{i=\overline{1, N}},\left(\theta_{j}\right)_{j=\overline{1, K}}\right) \in Y$ is indeed a weakly efficient solution of (MD).

Theorem 4.4. (Strict Converse Duality). Let $x_{0}$ and $\left(y_{0}, \lambda, \mu,\left(\eta_{i}\right)_{i=1, \mathrm{~N}},\left(\theta_{j}\right)_{j=1, \mathrm{k}}\right)$ be feasible solutions for (MP) and (MD) respectively, such that

$$
\sum_{i=1}^{N} \lambda_{i} f_{i}\left(x_{0}\right)=\sum_{i=1}^{N} \lambda_{i} f_{i}\left(y_{0}\right) .
$$

Moreover, assume that $(f, g)$ is strictly pseudo quasi $d_{I}-V$-type I at $y_{o}$ with respect to $\left(\eta_{i}\right)_{i=\overline{1, N}},\left(\theta_{j}\right)_{j=1, \bar{k}}$ and for $\lambda$ and $\mu$. Then $x_{0}=y_{0}$.

Proof. Since $\mu_{j} g_{j}\left(y_{0}\right) \geqq 0 \forall j=1,2, \cdots, k$, we have 


$$
b_{1}\left(x_{0} y_{0}\right) \phi_{1}\left[\sum_{j=1}^{k} \mu_{j} \beta_{j}\left(x_{0}, y_{0}\right) g_{j}\left(y_{0}\right)\right] \geqq 0 .
$$

Using the second part of the hypothesis, we get

$$
\sum_{j=1}^{k} \mu_{j} g_{j}^{\prime}\left(y_{0} ; \theta_{j}\left(x_{0}, y_{0}\right)\right) \leqq 0 .
$$

The Inequality (28) and feasibility of

$$
\begin{array}{r}
\left(y_{0}, \lambda, \mu,\left(\eta_{i}\right)_{i=\overline{1, N}},\left(\theta_{j}\right)_{j=\overline{1, k}}\right) \text { for (MD) give } \\
\sum_{i=1}^{N} \lambda_{i} f_{i}^{\prime}\left(y_{0} ; \eta_{i}\left(x_{0}, y_{0}\right)\right) \geqq 0,
\end{array}
$$

which by the first part of Hypothesis ii), we obtain

$$
\begin{aligned}
& b_{0}\left(x_{0}, y_{0}\right) \phi_{0}\left[\sum_{i=1}^{N} \lambda_{i} \alpha_{i}\left(x_{0}, y_{0}\right)\left(f_{i}\left(x_{0}\right)-f_{i}\left(y_{0}\right)\right)\right]>0, \\
& \forall x \in X .
\end{aligned}
$$

The above inequality along with Hypothesis iii) gives

$$
\sum_{i=1}^{N} \lambda_{i} \alpha_{i}\left(x_{0}, y_{0}\right)\left(f_{i}\left(x_{0}\right)-f_{i}\left(y_{0}\right)\right)>0 \text {. }
$$

By Hypothesis i), iii) and $\alpha_{i}\left(x_{0}, y_{0}\right)>0$, $i=1,2, \cdots, N$ we have

$$
\sum_{i=1}^{N} \lambda_{i} \alpha_{i}\left(x_{0}, y_{0}\right)\left(f_{i}\left(x_{0}\right)-f_{i}\left(y_{0}\right)\right)=0 .
$$

Now (29) and (30) contradict each other. Hence the conclusion follows.

\section{Conclusion and Future Developments}

In this paper, generalized $d_{I}-V$-univex functions have been introduced. The sufficient optimality conditions are discussed for a point to be an efficient or properly efficient for (MP) under the introduced functions. Appropriate Mond-Weir type duality relations are established under these assumptions. Sufficiency and duality with generalized $d_{I}-V$-univex functions will be studied for nonsmooth variational and nonsmooth control problems, which will orient the future research of the author.

\section{References}

[1] M. A. Hanson, "On Sufficiency of the Kunn-Tucker Conditions," Journal of Mathematical Analysis and Applications, Vol. 80, 1981, pp. 445-550.

[2] B. D. Craven, "Invex Functions and Constrained Local Minima,” Bulletin of Australian Mathematical Society, Vol. 24, No. 3, 1981, pp. 357-366. doi:10.1017/S0004972700004895

[3] R. N. Kaul and K. Kaur, "Optimality Criteria in Nonlinear Programming Involving Non Convex Functions,”
Journal of Mathematical Analysis and Applications, Vol. 105, No. 1, January 1985, pp. 104-112. doi:10.1016/0022-247X(85)90099-X

[4] M. A. Hanson and B. Mond, "Necessary and Sufficient Conditions in Constrained Optimization,” Mathematical Programming, Vol. 37, No. 1, 1987, pp. 51-58. doi:10.1007/BF02591683

[5] N. G. Ruedo and M. A. Hanson, “Optimality Criteria in Mathematical Programming Involving Generalized Invexity," Journal of Mathematical Analysis and Applications, Vol. 130, No. 2, 1988, pp. 375-385. doi:10.1016/0022-247X(88)90313-7

[6] F. Zhao, "On Sufficiency of the Kunn-Tucker Conditions in Non Differentiable Programming," Bulletin Australian Mathematical Society, Vol. 46, No. 3, 1992, pp. 385-389.

[7] F. H. Clarke, "Optimization and Nonsmooth Analysis," John Wiley and Sons, New York, 1983.

[8] R. N. Kaul, S. K. Suneja and M. K. Srivastava, “Optimality Criteria and Duality in Multi Objective Optimization Involving Generalized Invexity,” Journal of Optimization Theory and Applications, Vol. 80, No. 3, 1994, pp. 465482. doi:10.1007/BF02207775

[9] S. K. Suneja and M. K. Srivastava, "Optimality and Duality in Non Differentiable Multi Objective Optimization Involving $d$-Type I and Related Functions,” Journal of Mathematical Analysis and Applications, Vol. 206, 1997, pp. 465-479. doi:10.1006/jmaa.1997.5238

[10] H. Kuk and T. Tanino, "Optimality and Duality in Nonsmooth Multi Objective Optimization Involving Generalized Type I Functions," Computers and Mathematics with Applications, Vol. 45, No. 10-11, 2003, pp. $1497-$ 1506. doi:10.1016/S0898-1221(03)00133-0

[11] T. R. Gulati and D. Agarwal, "Sufficiency and Duality in Nonsmooth Multiobjective Optimization Involving Generalized $(F, \alpha, \rho, d)$-Type I Functions,” Computers and Mathematics with Applications, Vol. 52, No. 1-2, July 2006, pp. 81-94. doi:10.1016/j.camwa.2006.08.006

[12] R. P. Agarwal, I. Ahmad, Z. Husain and A. Jayswal, "Optimality and Duality in Nonsmooth Multiobjective Optimization Involving Generalized V -Type I Functions,” Journal of Inequalities and Applications, 2010. doi:10.1155/2010/898626

[13] A. Jayswal, I. Ahmad and S. Al-Homidan, "Sufficiency and Duality for Nonsmooth Multiobjective Programming Problems Involving Generalized $(F, \alpha, \rho, d)-d-V$ Univex Functions," Mathematical and Computer Modelling, Vol. 53, 2011, pp. 81-90. doi:10.1016/j.mcm.2010.07.020

[14] T. Antczak, "Multiobjective Programming under $d$-Invexity,” European Journal of Operational Research, Vol. 137, No. 1, 2002, pp. 28-36. doi:10.1016/S0377-2217(01)00092-3

[15] S. K. Mishra, S. Y. Wang and K. K. Lai, “Optimality and Duality in Nondifferentiable and Multi Objective Programming under Generalized d -Invexity,” Journal of Global Optimization, Vol. 29, No. 4, 2004, pp. 425-438. 
doi:10.1023/B:JOGO.0000047912.69270.8c

[16] S. K. Mishra, S. Y. Wang and K. K. Lai, "Nondifferentiable Multiobjective Programming under Generalized $d$ Univexity," European Journal of Operational Research, Vol. 160, No. 1, 2005, pp. 218-226. doi:10.1016/S0377-2217(03)00439-9

[17] S. K Mishra and M. A. Noor, "Some Nondifferentiable Multiobjective Programming Problems," Journal of Mathematical Analysis and Applications, Vol. 316, No. 2, April 2006, pp. 472-482. doi:10.1016/j.jmaa.2005.04.067

[18] Y. L. Ye, “ $d$-Invexity and Optimality Conditions,” Journal of Mathematical Analysis and Applications, Vol. 162, No. 1, November 1991, pp. 242-249. doi:10.1016/0022-247X(91)90190-B

[19] T. Antczak, "Optimality Conditions and Duality for Nondifferentiable Multi Objective Programming Problems Involving $d-r$-Type I Functions,” Journal of Computational and Applied Mathematics, Vol. 225, No. 1, March 2009, pp. 236-250. doi:10.1016/j.cam.2008.07.028

[20] H. Silmani and M. S. Radjef, "Nondifferentiable Multiobjective Programming under Generalized $d_{I}$-Invexity,” European Journal of Operational Research, Vol. 202,
2010, pp. 32-41. doi:10.1016/j.ejor.2009.04.018

[21] R. P. Agarwal, I. Ahmad and S. Al-Homidan, “Optimality and Duality for Nonsmooth Multiobjective Programming Problems Involving Generalized $d-\rho-(\eta, \theta)$-Type I Invex Functions,” Journal of Nonlinear and Convex Analysis, 2011.

[22] T. Antczak, "Mean Value in Invexity Analysis," Nonlinear Analysis: Theory, Methods and Applications, Vol. 60, No. 8, March 2005, pp. 1473-1484.

[23] A. Ben-Israel and B. Mond, "What is Invexity?" The Journal of Australian Mathematical Society Series B, Vol. 28, No. 1, 1986, pp. 1-9. doi:10.1017/S0334270000005142

[24] V. Jeyakumar and B. Mond, "On Generalized Convex Mathematical Programming," Journal of the Australian Mathematical Society Series B, Vol. 34, 1992, pp. 43-53. doi:10.1017/S0334270000007372

[25] M. A. Hanson, R. Pini and C. Singh, "Multiobjective Programming under Generalized Type I Invexity,” Journal of Mathematical Analysis and Applications, Vol. 261, No. 2, September 2001, pp. 562-577. doi:10.1006/jmaa.2001.7542 